\title{
Anti-phospholipid IgG antibodies detected by line immunoassay differentiate patients with anti-phospholipid syndrome and other autoimmune diseases
}

\author{
Cecilia Nalli ${ }^{1}$. Valentina Somma ${ }^{2}$ Laura Andreoli ${ }^{1} \cdot$ Thomas Büttner $^{3} \cdot$ Peter Schierack ${ }^{4} \cdot$ Michael Mahler $^{5}$. \\ Dirk Roggenbuck ${ }^{2,4}$ (D) Angela Tincani ${ }^{1}$
}

Received: 6 May 2018 / Accepted: 14 May 2018 / Published online: 29 May 2018

(c) The Author(s) 2018

\begin{abstract}
Purpose Anti-phospholipid antibodies (aPL) analyzed by line immunoassay (LIA) can recognize beta ${ }_{2}$-glycoprotein I ( $\beta_{2}$ GPI) domain 1 (D1) epitopes depending on $\beta_{2}$ GPI binding to distinct phospholipids. The aPL LIA was compared with consensus ELISA to investigate whether both techniques can discriminate anti-phospholipid syndrome (APS) patients from aPLpositive, systemic autoimmune rheumatic diseases (SARD) patients without clinical symptoms of APS and controls.

Methods Thirty-four APS patients (14 arterial/venous thrombosis, 16 pregnancy morbidity, and 4 both), 41 patients with SARD lacking clinical APS criteria but demonstrating positivity for anti- $\beta_{2}$ GPI (a $\beta_{2}$ GPI) IgG, and 20 healthy subjects (HS) were tested for aPL to cardiolipin (aCL), phosphatidic acid, phosphatidylcholine, phosphatidylethanolamine, phosphatidylglycerol (aPG), phosphatidylinositol, phosphatidylserine, $\beta_{2}$ GPI, prothrombin, and annexin V by LIA. Samples were also tested for $\mathrm{aCL}, \mathrm{a} \beta_{2}$ GPI, a $\beta_{2}$ GPI-domain 1 (aD1), and a $\beta_{2}$ GPI-domains 4-5 (aD4-5) by ELISA and for lupus anti-coagulant. Results Comparison of LIA with ELISA revealed a good agreement for the consensus criteria aPL a $\beta_{2}$ GPI and aCL IgG (kappa $=0.69,0.68$, respectively) and a moderate agreement for $\operatorname{IgM}$ (kappa $=0.52,0.49$, respectively). Regarding ELISA, aD1/aD4-5 demonstrated the best performance of differentiating APS from asymptomatic SARD [area under the curve (AUC): 0.76]. aPG IgG had the best performance by LIA (AUC: 0.72) not significantly different from aD1/aD4-5. There was a good agreement for $\mathrm{aPG}$ IgG with $\mathrm{aD} 1 / \mathrm{aD} 4-5$ (kappa $=0.71)$.

Conclusions aD1/aD4-5 (ELISA) and aPG IgG (LIA) differentiate APS from SARD patients. PG appears to interact with $\beta_{2}$ GPI of APS patients and exposes D1 thereof for disease-specific aPL binding in LIA.
\end{abstract}

Keywords Anti-phospholipid syndrome - Beta2 glycoprotein I · Anti-phospholipid antibody · Domain 1 .

Phosphatidylglycerol

\section{Introduction}

Anti-phospholipid syndrome (APS) is an autoimmune disorder, clinically characterized by arterial and/or venous thrombosis as well as pregnancy-related complications $[1,2]$. The APS can be primary or secondary, depending on the absence or presence of any other related systemic autoimmune

Cecilia Nalli and Valentina Somma shared first authorship.

Dirk Roggenbuck and Angela Tincani shared senior authorship.

Dirk Roggenbuck

dirk.roggenbuck@b-tu.de

Extended author information available on the last page of the article rheumatic disease (SARD) such as, e.g., systemic lupus erythematosus (SLE). The APS could be associated with a high risk of death in the rare catastrophic anti-phospholipid syndrome, a rapid and simultaneous multi-organ failure due to generalized thrombosis [3]. Apart from one clinical criterion (vascular thrombosis and/or adverse obstetric event), the revised classification criteria require the persistent detection of anti-phospholipid antibodies (aPL) such as anti-beta $_{2}$ glycoprotein I ( $a \beta_{2}$ GPI), anti-cardiolipin ( $\mathrm{aCL}$ ), and/or autoantibodies interfering with coagulation [lupus anti-coagulant (LAC)] for the diagnosis of APS.

The recommended method to detect $\mathrm{a} \beta_{2}$ GPI and $\mathrm{aCL}$ is the enzyme-linked immunosorbent assay (ELISA) using a polystyrene solid phase for autoantigen immobilization. However, aPL testing by ELISA still represents a challenge because of 
the difficulties in the inter- and intra-assay reproducibility [4, 5]. Furthermore, a $\beta_{2}$ GPI antibodies detected by ELISA have been reported in healthy adults and children. These data support the hypothesis that "innocent", non-disease associated aPL could exist, too $[6,7]$. The subgroup of pathogenic $a \beta_{2}$ GPI antibodies seems to be mainly directed versus domain 1 (D1) and not to domains 4 and 5 (D4-5), and their pathogenicity appears to be dependent on their Fc glycosylation [8-12]. Indeed, the former are involved in thrombotic events characteristic of APS, whereas the latter do not interfere with the coagulation process, neither are they associated with other clinical APS manifestations [13].

New assay techniques based on chemiluminescence (CIA) or fluorescence enzyme immunoassays for the detection of APS-specific aPL have emerged [4, 14]. Especially, the CIA system has been shown to reduce the inter-laboratory variability [15].

Of note, a novel line immunoassay (LIA) offering the opportunity to test for several aPL has been reported [16, 21]. This LIA appeared to detect preferably aPL to $\mathrm{D} 1 \mathrm{aD} 1)$ of the patient's $\beta_{2}$ GPI bound to distinct negatively charged phospholipids. Furthermore, aPL not related to a $\beta 2$ GPI were detected, too [16]. Altogether, the former and these "non-criteria" IgG and IgM aPL to phosphatidylserine (aPS), phosphatidylinositol (aPI), phosphatidylcholine (aPC), phosphatidylethanolamine (aPE), phosphatidic acid (aPA), phosphatidylglycerol (aPG), annexin $\mathrm{V}(\mathrm{aAnV})$, and prothrombin (aPT) could be used for aPL profiling and might be helpful in the clinical differentiation of APS patients [17-20]. Nevertheless, the clinical meaning of "non-criteria" antibodies is still debated and $\beta_{2}$ GPI is generally accepted as the major autoantigenic target recognized by APS-specific aPL [2].

The novel LIA used a hydrophobic membrane for the immobilization of different phospholipids and co-factors [21, 22] (Fig. 1). In particular, negatively charged phospholipids could bind the patient's own $\beta 2$ GPI which in turn interacted with APS-specific aD1. Thus, the specificity of this new multiplex reaction environment was reported to be superior to aPL consensus ELISA [23]. In a recent study comparing aPL testing by LIA with ELISA in APS patients, asymptomatic aPLpositive carriers, and infectious patients, the LIA demonstrated a better specificity, too [16].

The appearance of aPL in SARD patients without characteristic clinical signs of APS is poorly understood yet. Thus, we wondered whether aPL detected by LIA or consensus criteria ELISA could discriminate primary APS from SARD without clinical symptoms of APS.

\section{Methods}

\section{Patients and control subjects}

Thirty-four patients with primary APS including 14 with arterial and/or venous thrombosis, 16 females with obstetric APS suffering from pregnancy-related complications, and 4 having both clinical symptoms were diagnosed by characteristic international clinical and serological consensus criteria (Table 1). The patients were selected from a cohort routinely followed at the university hospital in Brescia. All patients demonstrated elevated levels of a $\beta_{2}$ GPI IgG antibodies by an in-house ELISA. This inclusion criterion was chosen to study the specificity of these antibodies against different $\beta_{2}$ GPI domains.

As disease controls, 41 patients with SARD and no anamnestic thrombotic and adverse pregnancy events but positivity for a $\beta_{2}$ GPI IgG [11 with SLE, 2 with systemic sclerosis ( $\mathrm{SSc}$ ), 2 with Sjögren syndrome (SjS), 3 with SLE and secondary $\mathrm{SjS}, 15$ with undifferentiated connective tissue disease (UTCD), 1 with discoid lupus erythematosus (DLE), 4 with dermato/polymyositis (DM/PM), and 3 patients with primary biliary cholangitis (PBC)] were enrolled. This group was chosen due to the comparability with the disease group. Furthermore, 20 healthy subjects (children) (HS) were included as non-diseased controls. All children were aPL negative. The study was approved by the local ethical committee after a written informed consent from each patient. All sera were stored at $-20^{\circ} \mathrm{C}$.

\section{ELISA for the detection of antibodies to cardiolipin and $\beta_{2}$ GPI}

To detect classification criteria $\operatorname{IgG}$ and $\operatorname{IgM}$ antibodies to $\mathrm{CL}$ and $\beta_{2}$ GPI in the patient sera, commercially available solid-phase ELISAs employing purified human $\beta_{2}$ GPI in complex with CL and human $\beta_{2}$ GPI alone were used, respectively (GA Generic Assays GmbH, Dahlewitz, Germany). Assessment of aPL antibodies was conducted according to the instructions of the manufacturer [21]. The sera with a concentration equal or more than $10 \mathrm{U} / \mathrm{mL}$ for $\mathrm{IgG}$ and IgM, respectively, was considered positive. The same serum samples were also analyzed by in-house assays and the results were comparable with the commercial ELISA (data not shown).

Research ELISAs for aD1 and aD4-5 IgG developed by Inova Diagnostics (San Diego, US) were performed as previously described [9]. A ratio of aD1 and aD4 -5 with a cutoff of 1.5 was used to test sera for aPL positivity. 
A

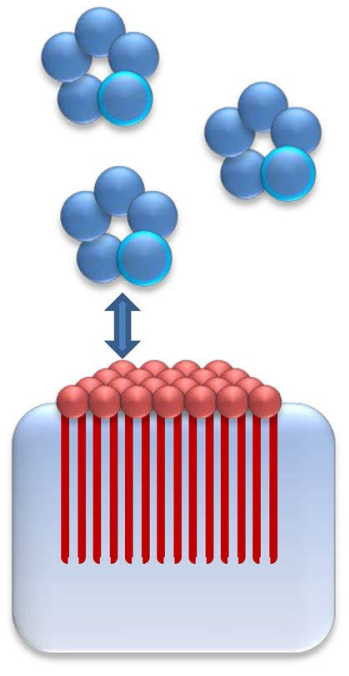

B
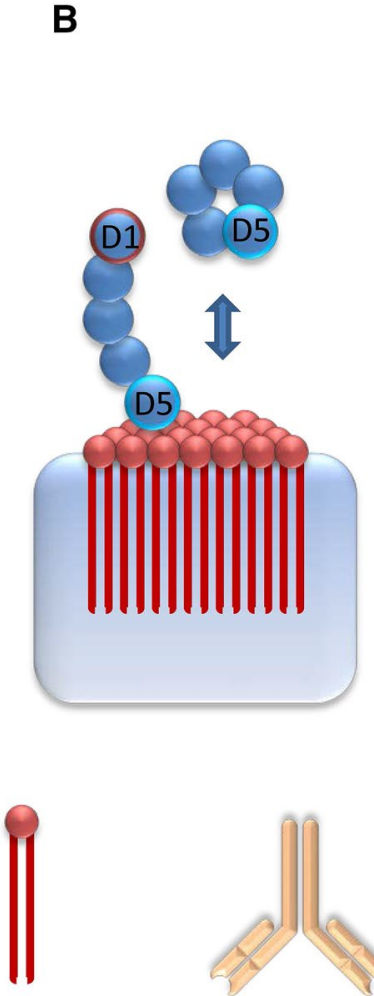

$\mathrm{PL}$

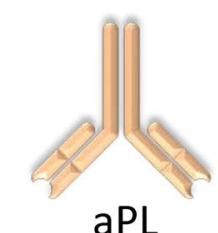

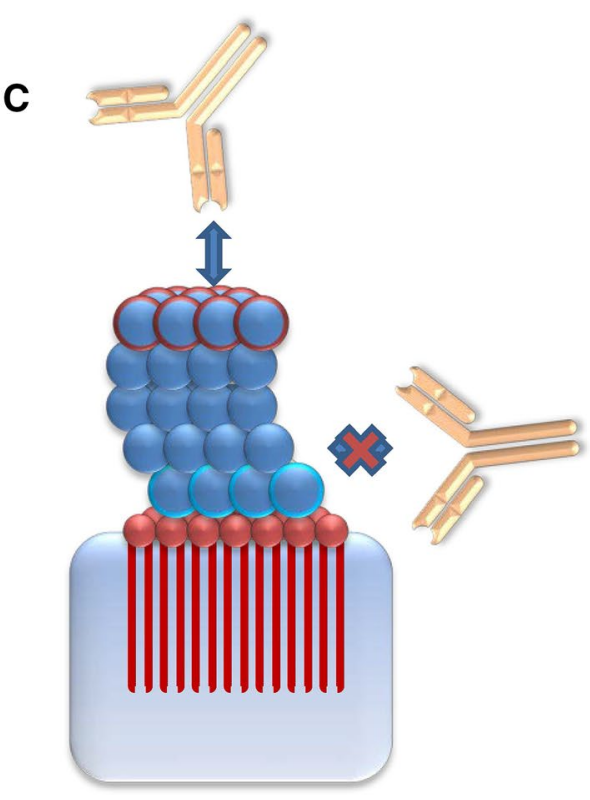

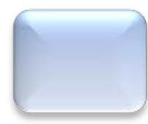

membrane
Fig. 1 Preferential binding of anti-phospholipid antibodies (aPL) to domain 1 (D1) of patient's beta2-glycoprotein I ( $\left.\beta_{2} \mathrm{GPI}\right)$ in the line immunoassay (LIA). In contrast to the planar solid phase used in enzyme immunoassays, the porous hydrophobic LIA membrane incorporates the hydrophobic phospholipid (PL)-tail during immobilization. This shields the by far larger tail of the amphiphatic PL molecule from the reaction environment and, thus, prevents unspecific interactions. Number, orientation, and accessibility of anionic phosphate groups of the differing hydrophilic PL heads may influence the binding of the patient's $\beta_{2}$ GPI (a) and consequently of the $\beta_{2}$ GPIdependent aPL (c). After binding of $\beta_{2}$ GPI to the immobilized anionic PL by domain 5 (D5, containing the PL-binding site), D1 forms the accessible top of the induced fish-hook-like $\beta_{2}$ GPI structure (b). Due to the high density of negatively charged PL heads on the membrane, the formation of a $\beta_{2}$ GPI layer with a unique D1 epitope structure is assumed. The layer formation seems to hinder aPL binding to $\beta_{2}$ GPI epitopes close to D5 [16]

\section{LAC testing}

The analysis of lupus anti-coagulant (LAC) was performed according to the international recommendations [24]. Thus, the LAC testing comprised a three-step procedure:

- Demonstration of a prolonged phospholipid-dependent clotting time as screening test of hemostasis by dilute Russell viper venom time (dRVVT) or activated partial thromboplastin time (aPTT or lupus aPTT) analysis.

- Mixing patient plasma with normal plasma fails to correct the prolonged screening test(s).

- Addition of excess phospholipid shortens or corrects the prolonged coagulation test (demonstration of phospholipid dependence).

\section{Line immunoassay for the detection of aPL antibodies}

Antibodies to CL, PA, PC, PE, PG, PI, PS, the protein cofactors $\beta_{2} \mathrm{GPI}, \mathrm{AnV}$, and PT were detected using a commercially available LIA according to the recommendations of the manufacturer (GA Generic Assays GmbH) [16]. Processed LIA strips were read out densitometrically employing a scanner with the evaluation software Dr. Dot Line Analyzer (GA Generic Assays $\mathrm{GmbH}$ ) and a grayscale calibration card for standardization. The grayscale calibration card was provided on the template of the kit. Values were read off as optical density (OD) units and OD values equaling or above 50 were scored positive. This cutoff was determined by calculating the 99 th percentile of 150 apparently healthy 
Table 1 Demographic, clinical, and laboratory baseline characteristics of the 75 anti-beta 2 glycoprotein I (a $\beta_{2}$ GPI) IgG-positive patients and 20 healthy subjects (children)

\begin{tabular}{|c|c|c|c|}
\hline & $\begin{array}{l}\text { Primary thrombotic or obstet- } \\
\text { ric APS }(n=34)\end{array}$ & $\operatorname{SARD}(n=41)$ & Healthy subjects $(n=20)$ \\
\hline Sex, no. $(\%)$ female & $31 / 34(91 \%)$ & $36 / 41(88 \%)$ & $9 / 20(55 \%)$ \\
\hline Autoimmune disease & $34 / 34(100 \%)$ & $41 / 41(100 \%)$ & $0(0 \%)$ \\
\hline Primary APS & $34 / 34(100 \%)$ & $0(0 \%)$ & $0(0 \%)$ \\
\hline SLE & $0(0 \%)$ & $11 / 41(27 \%)$ & $0(0 \%)$ \\
\hline $\mathrm{SSj}$ & $0(0 \%)$ & $2 / 41(5 \%)$ & $0(0 \%)$ \\
\hline $\mathrm{SLE}+\mathrm{SSj}$ & $0(0 \%)$ & $3 / 41(7 \%)$ & $0(0 \%)$ \\
\hline DLE & $0(0 \%)$ & $1 / 41(2 \%)$ & $0(0 \%)$ \\
\hline PBC & $0(0 \%)$ & $3 / 41(7 \%)$ & $0(0 \%)$ \\
\hline $\mathrm{SSc}$ & $0(0 \%)$ & $2 / 41(5 \%)$ & $0(0 \%)$ \\
\hline $\mathrm{DM} / \mathrm{PM}$ & $0(0 \%)$ & $4 / 41(10 \%)$ & $0(0 \%)$ \\
\hline UCTD & $0(0 \%)$ & $15 / 41(37 \%)$ & $0(0 \%)$ \\
\hline Thrombosis & $19 / 34(56 \%)$ & $0(0 \%)$ & $0(0 \%)$ \\
\hline Arterial & $7 / 19(37 \%)$ & $0(0 \%)$ & $0(0 \%)$ \\
\hline Venous & $12 / 19(63 \%)$ & $0(0 \%)$ & $0(0 \%)$ \\
\hline Obstetric manifestations & $20 / 34(59 \%)$ & $0(0 \%)$ & NA \\
\hline Pregnancy loss & $15 / 20(75 \%)$ & $0(0 \%)$ & NA \\
\hline Preeclampsia & $5 / 20(25 \%)$ & $0(0 \%)$ & NA \\
\hline \multicolumn{4}{|l|}{ Laboratory features } \\
\hline LAC positivity & $24 / 34(71 \%)$ & $18 / 41(44 \%)$ & $\mathrm{NP}$ \\
\hline $\mathrm{a} \beta_{2} \mathrm{GPI}$ IgG, median OD (25-75th percentile) & $1.470(0.929-1.747)$ & $1.004(0.655-1.298)$ & $0.139(0.047-0.444)$ \\
\hline $\mathrm{a} \beta_{2}$ GPI IgM, median OD (25-75th percentile) & $0.350(0.165-0.576)$ & $0.450(0.202-0.838)$ & $0.088(0.049-0.132)$ \\
\hline
\end{tabular}

$A P S$ anti-phospholipid syndrome, $a C L$ anti-cardiolipin antibodies, $D L E$ discoid lupus erythematosus, $D M / P M$ dermato/polymyositis, $L A C$ lupus anti-coagulant, $N A$ not applicable, $N P$ not performed, $O D$ optical density, $P B C$ primary biliary cirrhosis, $S A R D$ systemic autoimmune rheumatic disease, $S L E$ systemic lupus erythematosus, $S S c$ systemic scleroderma, SjS Sjögren syndrome, UCTD undifferentiated connective tissue disease

individuals as recommended by the international classification criteria for aPL testing and Clinical and Laboratory Standards Institute (CLSI) guideline C28-A3 [25].

\section{Statistical analysis}

Fisher's exact test was performed with two-tailed probability to detect the differences between groups as appropriate using Medcalc statistical software (Medcalc, Mariakerke, Belgium). Inter-rater agreement statistics ware applied for within-group comparison. The two-tailed, Kruskal-Wallis test was used to test for statistically significant differences of independent samples. $p$ values of less 0.05 were considered significant.

\section{Results}

\section{Comparison of aPL analysis by ELISA and LIA}

To identify the aPL antibody profiles by ELISA and LIA, we tested 34 sera from patients with APS and 61 controls including 41 asymptomatic patients suffering from SARD and $20 \mathrm{HS}$ (Table2). Comparative analysis of the consensus criteria aPL aCL and $a \beta_{2}$ GPI in 95 sera detected by LIA and ELISA demonstrated good agreement for IgG [kappa $=0.69$, 95\% confidence interval (CI) $0.55-0.84 ; 0.68,95 \%$ CI $0.54-0.83$, respectively] and moderate concordance for $\operatorname{IgM}$ $(\mathrm{kappa}=0.52,95 \%$ CI 0.35-0.69; 0.49, 95\% CI 0.32-0.66, respectively). There was a significant difference according to McNemar's test for aCL and a $\beta_{2}$ GPI IgM (difference: 15.8 and $13.7 \%, p<0.05$, respectively), whereas the corresponding IgG analyses did not reveal a significant difference for both methods. The consensus ELISA testing for aCL and a $\beta_{2}$ GPI is not significantly more specific than aPL analysis by LIA covering $10 \mathrm{aPL}$ with regard to the false positives in HS $(1 / 20$ vs $3 / 20, p=0.605)$.

\section{Comparison of aPL testing in APS patients and healthy controls}

Both LIA and ELISA showed significantly higher prevalences of positive consensus criteria aPL (aCL and a $\beta_{2}$ GPI IgG as well as $\operatorname{IgM})$ in APS patients $(n=34)$ compared to 
HS $(n=20)(p<0.05$, respectively, Table 2$)$. In addition, the LIA revealed significantly more prevalent aPA and aPS IgG as well as IgM, and further $\mathrm{aPG} \operatorname{IgG}$, aPI IgG, and aPT IgG in APS patients ( $p<0.05$, respectively).

\section{Comparison of qualitative aPL testing in APS patients and disease controls}

The comparison of APS patients $(n=34)$ with asymptomatic SARD patients $(n=41)$ revealed significantly higher prevalences in APS patients for the criteria aPL aCL and $\mathrm{a} \beta_{2} \mathrm{GPI}$ IgG detected by ELISA $(p<0.05$, respectively, Table 2$)$. The, frequency of LAC positivity was also significantly elevated in APS, whereas the frequency of triple positivity demonstrated a tendency only $(p=0.0312,0.0713$, respectively). In terms of aPL testing by LIA, aPG IgG, a $\beta_{2}$ GPI $\mathrm{IgG}$, and aPT IgG as well as aPS IgG and IgM were significantly higher prevalent in patients suffering from APS in contrast to SARD patients. Of note, aPG IgG showed a significantly lower prevalence of $9.8 \%$ in asymptomatic SARD patients (4/41) compared to $52.9 \%$ in APS patients $(18 / 34, p<0.0001)$. The same holds true for aPT IgG with a prevalence of $17.1 \%$ in SARD (7/41) vs $50.0 \%$ in APS $(17 / 34, p=0.0002)$.

\section{Comparison of quantitative aPL testing in APS patients and controls}

Quantitative assessment revealed significantly different aPL IgG and IgM levels in the study cohorts regarding all consensus criteria aPL ( $\mathrm{aCL}$ and $\mathrm{a} \beta_{2} \mathrm{GPI}$ ) by ELISA (Kruskal-Wallis, $p<0.05$, respectively (Fig. 2). In terms of LIA testing, IgG and IgM to CL, $\beta_{2}$ GPI, PA, PS, PT, and PG as well as IgM to AnV demonstrated significantly different values (Kruskal-Wallis, $p<0.05$, respectively).

Regarding the differentiation of APS patients from asymptomatic SARD patients by consensus criteria ELISA, only aCL and $\mathrm{a} \beta_{2}$ GPI IgG revealed significantly different quantitative levels (post hoc analysis, $p<0.05$, respectively) (Fig. 2). In addition to aCL and aß2GPI IgG detected by LIA, IgG to PA, PS, PG, PT, and IgM to PS revealed significantly higher levels in APS patients, too (post hoc analysis, $p<0.05$, respectively) (Fig. 3).

\section{Comparison of the assay performance of aPL detected by ELISA and LIA}

To compare the diagnostic performance for the differentiation of APS from SARD, receiver-operating characteristic (ROC) curve analysis was performed for aPL detected by ELISA and LIA (Fig. 4). The ratio of D1 to D4-5 reactivity demonstrated the best performance with an area under the curve (AUC) of 0.76 when compared with consensus 

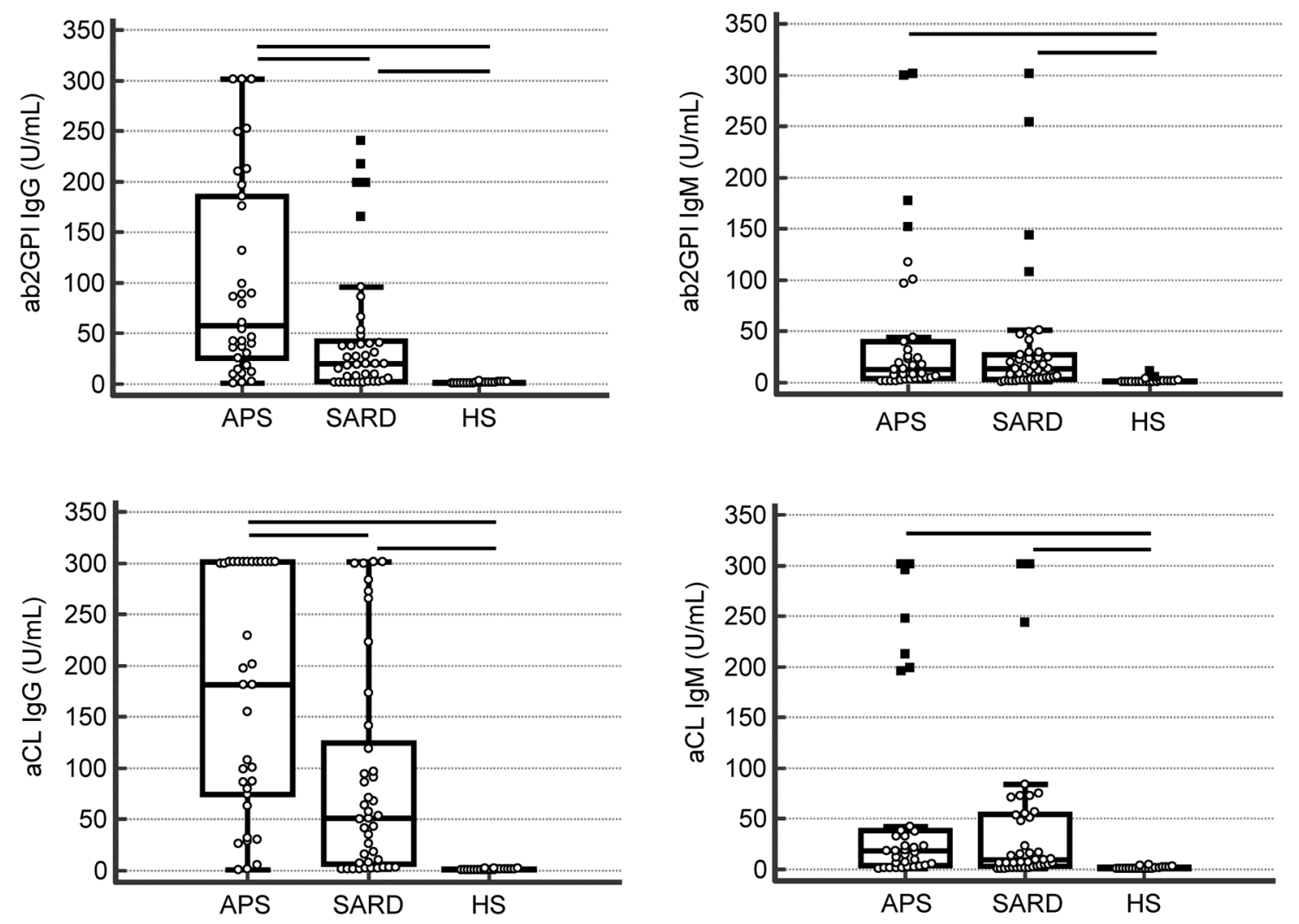

Fig. 2 Consensus criteria anti-phospholipid antibodies detected by enzyme-linked immunosorbent assays (ELISAs) in 95 subjects including 34 patients with anti-phospholipid syndrome (APS), 41

criteria aPL ELISAs. The AUC of this ratio was significantly higher than the AUCs of aCL and a $\beta_{2}$ GPI IgM $(p<0.05$, respectively) (Table 3 ). However, there was no significant difference in the prevalence of the $\mathrm{aD} 1 / \mathrm{aD} 4-5$ ratio in APS in contrast to SARD using the cutoff of 1.5 established in another study previously [9]. In accordance with the ROC curve analysis for the aD1/aD4-5 ratios of this study, a cutoff of 4.6 instead of 1.5 for the optimal differentiation of APS and SARD was required. Applying this new cutoff, there were 21/34 (61.8\%) positive APS patients in contrast to $28 / 43$ (82.4\%) with the old cutoff of 1.5 . Accordingly, the new cutoff lowered the prevalence of positives in the asymptomatic SARD cohort from 26/41 (63.4\%) to 5/41 (12.2\%). Consequently, the new prevalence of the APS cohort was significantly higher in contrast to the one of the SARD cohorts $(p<0.0001)$.

Furthermore, there was no significant difference of the AUC for the aD1/aD4-5 ratio to the AUC of aPG IgG detected by LIA. The latter demonstrated in turn the best performance among the aPL IgG determined by LIA and was significantly higher than the AUC of aCL IgG (Table 3). In accordance with inter-rater agreement statistics, there was a good agreement for $\mathrm{aPG}$ IgG with the $\mathrm{aD} 1 / \mathrm{aD} 4-5$ ratio

with systemic autoimmune rheumatic disease (SARD) and no adverse APS events, as well as 20 healthy subjects (children) (HS) as control group. $a \beta_{2} G P I$ anti-beta 2 -glycoprotein I, $a C L$ anti-cardiolipin

$[\mathrm{kappa}=0.71,95 \%$ confidence interval (CI) 0.52-0.89] and no significant difference (McNemar's test: difference $=5.3 \%$, $95 \% \mathrm{CI}-6.8$ to $15.7 \%, p=0.4807)$.

In contrast, the strength of agreement of the $\mathrm{aD} 1 / \mathrm{aD} 4-5$ ratio with all four consensus criteria aPL determined by ELISA was only fair (kappa $<0.4$, aCL IgM, and a $\beta_{2}$ GPI $\mathrm{IgG}$ ) or poor (kappa $<0.2$, aß2GPI IgM, and $\mathrm{aCL}$ IgG).

\section{Discussion}

The persistent occurrence of aPL was the serological hallmark of APS and was defined as a mandatory classification criterion [26]. It is a well-accepted consensus that APSspecific aPL interact with phospholipid-binding proteins such as $\beta_{2}$ GPI or complexes thereof with phospholipids. Among aPL, the correlation of a $\beta_{2}$ GPI with clinical symptoms appeared to be the strongest one [2, 20, 27]. In this context, aPL binding to D1 and not to D4-5 of $\beta_{2}$ GPI has been the basis for the detection of disease-specific aPL [9, 28]. There has been no single assay to assess all different aPL subpopulations, and thus, aCL, a $\beta_{2}$ GPI, and LAC testing have been recommended to identify all the potential aPL. 

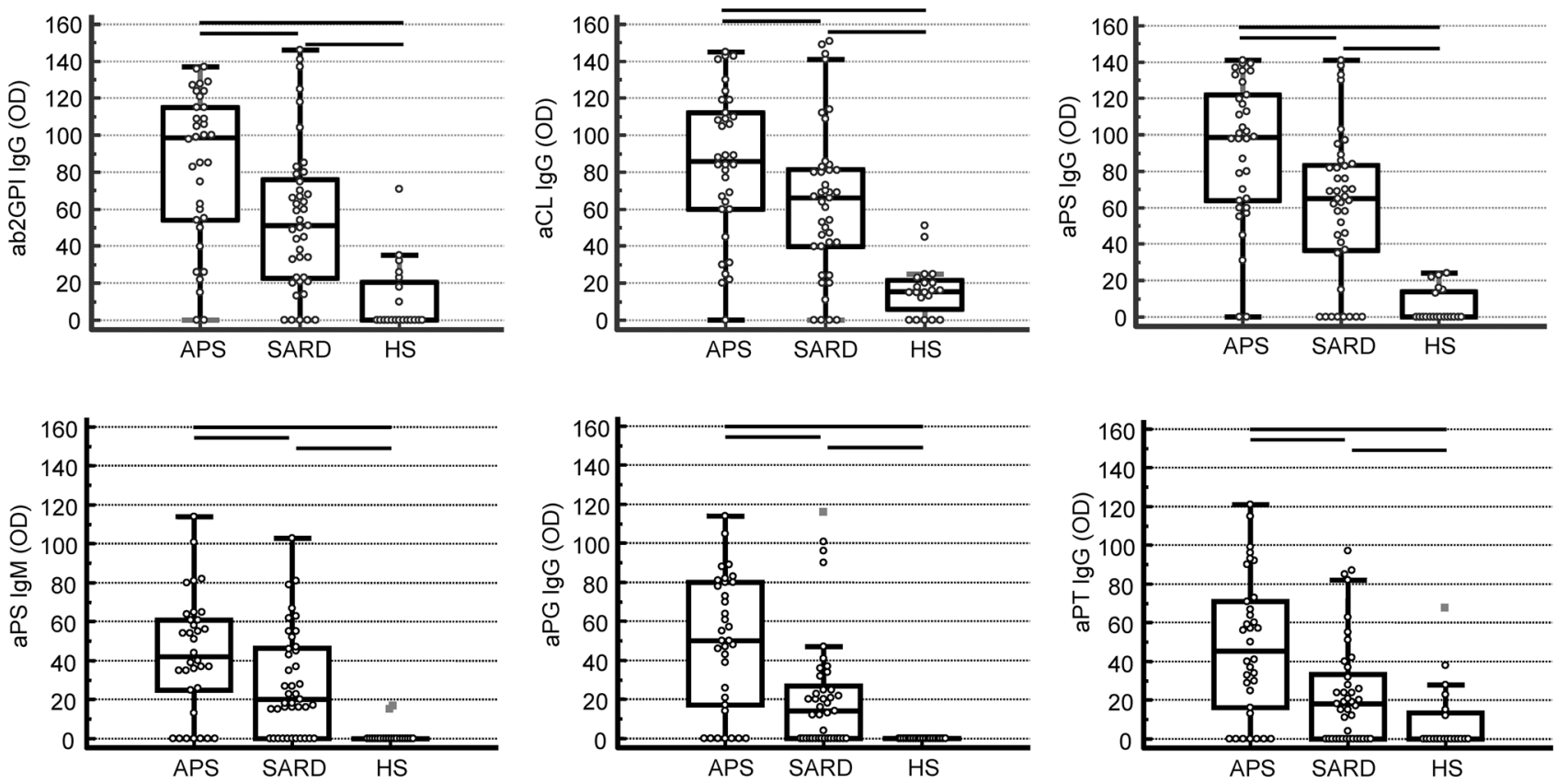

Fig. 3 Anti-phospholipid IgG antibodies analyzed by line immunoassay (LIA) in 95 subjects including 34 patients with anti-phospholipid syndrome (APS), 41 systemic autoimmune rheumatic disease (SARD), and no adverse APS events, as well as 20 healthy subjects

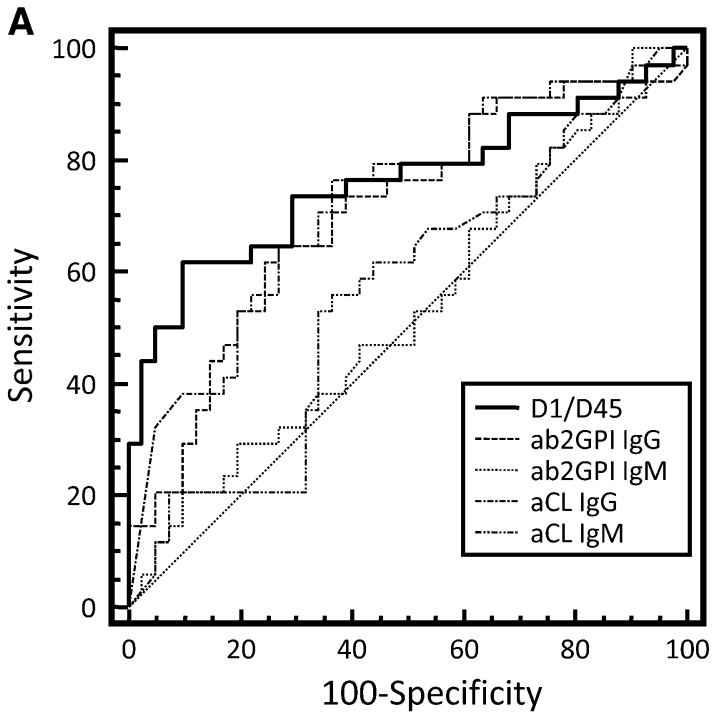

Fig. 4 Receiver-operating characteristics curve analysis of anti-phospholipid antibodies (aPL) detected by enzyme-linked immunosorbent assay (ELISA) and line immunoassay (LIA) in 34 patients with anti-phospholipid syndrome and 41 disease controls without clinical APS symptoms. The ratio of anti-domain $1(\mathrm{aD} 1)$ and D4-5 antibody (children) (HS) as control group. $a \beta_{2} G P I$ anti-beta ${ }_{2}$-glycoprotein I, $a C L$ anti-cardiolipin, $a P G$ anti-phosphatidylglycerol, $a P I$ anti-phosphatidylinositol, $a P S$ anti-phosphatidylserine, $a P T$ anti-prothrombin, $O D$ optical density

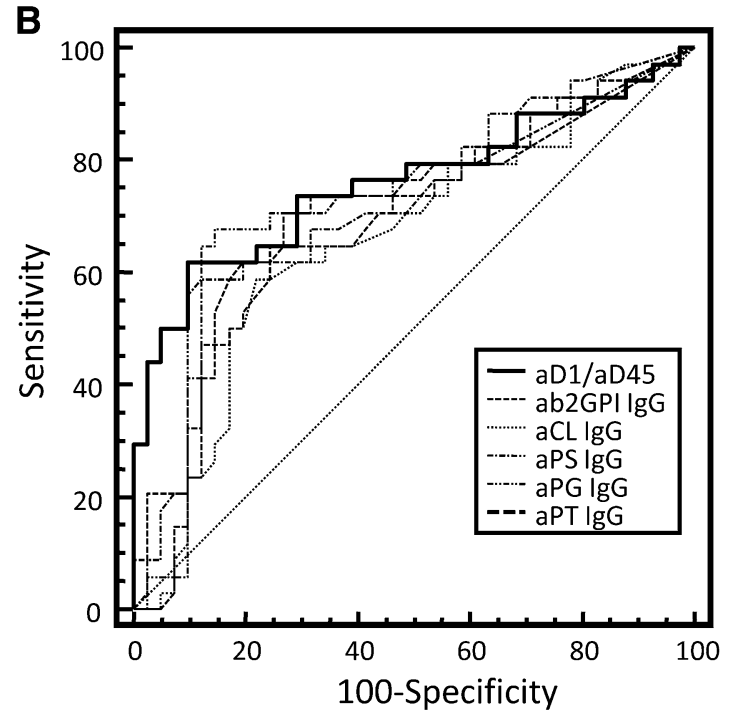

reactivity (aD4-5) by ELISA was compared with criteria aPL determined by ELISA (a) and aPL IgG by LIA (b). $a \beta_{2} G P I$ anti-beta ${ }_{2}-$ glycoprotein I, $a C L$ anti-cardiolipin, $a P G$ anti-phosphatidylglycerol, $a P I$ anti-phosphatidylinositol, $a P S$ anti-phosphatidylserine, $a P T$ antiprothrombin 
Table 3 Receiver-operating characteristics curve analysis of antiphospholipid antibodies (aPL) detected by enzyme-linked immunosorbent assay (ELISA) and line immunoassay (LIA) in 34 patients with anti-phospholipid syndrome and 41 disease controls

\begin{tabular}{llll}
\hline aPL & AUC & SE & $95 \%$ CI \\
\hline ELISA & & & \\
aD1/aD4-5 & 0.760 & 0.0597 & $0.647-0.851$ \\
a $\beta_{2}$ GPI IgG & 0.705 & 0.0617 & $0.588-0.805$ \\
a $\beta_{2}$ GPI IgM & $0.534^{*}, \S$ & 0.0679 & $0.416-0.651$ \\
aCL IgG & 0.725 & 0.0598 & $0.609-0.821$ \\
aCL IgM & $0.559^{*}, \S$ & 0.0679 & $0.440-0.674$ \\
LIA & & & \\
a $\beta_{2}$ GPI IgG & 0.691 & 0.0638 & $0.574-0.793$ \\
aCL IgG & 0.660 & 0.0651 & $0.541-0.765$ \\
aPS IgG & $0.716^{\&}$ & 0.0613 & $0.600-0.814$ \\
aPG IgG & $0.723^{\&}$ & 0.0630 & $0.608-0.821$ \\
aPT IgG & 0.701 & 0.0628 & $0.584-0.801$ \\
\hline
\end{tabular}

Area under the curve (AUC) was determined for the ratio of antidomain 1 of beta 2 glycoprotein I (aD1) and domains 4-5 (D4-5) antibody reactivity (D4-5) by ELISA and compared with those for criteria aPL determined by ELISA and aPL IgG by LIA

$a \beta_{2} G P I$ anti-beta 2 glycoprotein I, $a C L$ anti-cardiolipin, $a P G$ antiphosphatidyl-glycerol, $a P I$ anti-phosphatidylinositol, $a P S$ anti-phosphatidyl-serine, $a P T$ anti-prothrombin, $C I$ confidence interval, $S E$ standard error

AUC comparison of ELISA

${ }^{*} p<0.05$ for the comparison to the AUC of the ratio of aD1 to D4-5

${ }_{p} p<0.05$ for the comparison to the AUC of a $\beta_{2}$ GPI IgG

${ }^{\S} p<0.05$ for the comparison to the AUC of aCL IgG

AUC comparison of LIA

${ }^{\&} p<0.05$ for the comparison to the AUC of aCL IgG

Triple positivity has been considered a risk factor and could be used for stratification of APS patients [29].

A novel aPL assay technology employing a hydrophobic membrane for aPL profiling by LIA was reported recently [19]. The LIA membrane provided a unique matrix allowing phospholipids to mimic their natural conformation required for co-factor binding as reported for other amphiphatic nonprotein antigenic molecules [30-32]. In particular, D1 of patient's serum $\beta_{2}$ GPI appeared to be presented in the LIA reaction environment for APS-specific aPL binding more favorably than the corresponding D4-5 after the interaction of serum $\beta_{2}$ GPI with the immobilized phospholipids (Fig. 1). Interestingly, complexes of the patient's $\beta_{2}$ GPI with differing immobilized phospholipids demonstrated different $\mathrm{aD} 1$ reactivity. Altogether, this seemed to support the differentiation of disease-specific aPL in APS patients from aPL found in individuals with infectious disease or in asymptomatic carriers [16]. Thus, we attempted to ascertain whether this novel reaction environment for the multiplex detection of aPL can discriminate aPL in APS patients from those occurring in patients with other autoimmune disorders like SARD not demonstrating clinical symptoms of APS.

The agreement of aPL testing by LIA with consensus criteria aPL by ELISA was good (IgG aPL) to moderate (IgM aPL) and, thus, was in line with previously published comparative data [16, 21]. In addition, the favorable specificity of aPL testing by LIA could also be confirmed in this study revealing no significant difference for all ten aPL tested by LIA compared with the four consensus criteria aPL by ELISA.

In terms of the occurrence of aPL in APS patients compared with that in SARD patients without clinical symptoms of APS, only IgG consensus criteria aPL demonstrated a significant difference. Although LAC testing revealed significantly different prevalences too, triple positivity analysis did not differentiate APS patients from those with SARD. Of interest, aPL IgG by LIA also revealed significantly higher prevalences in APS compared to SARD without clinical symptoms of APS. In contrast to aCL IgG by ELISA, however, the difference of aCL IgG by LIA did not reach significance. Of note, aPS testing demonstrated for both immunoglobulin isotypes significantly different prevalences. Provided that the positive aPS result was due to interaction of patient's aPS with serum $\beta_{2}$ GPI of the patient sample having bound to immobilized PS on the LIA membrane, this might add further evidence to the assumption that $\beta_{2}$ GPI binding to negatively charged phospholipids induces specific conformational changes unique for each distinct phospholipid. Since D1 binding by a $\beta_{2}$ GPI was preferred in the LIA reaction environment [16], the differing accessibility of respective epitopes on D1 could determine the specificity of such aPL reactivity. Thus, the significantly reduced prevalence of aPG IgG in SARD without clinical symptoms of APS (9.8\%) compared to APS (52.9\%) in this study is of particular interest in this context. Of note, $\mathrm{CL}$ also referred to as diphosphatidylglycerol represents a dimer of PG and CL's head bears two phosphate groups forming a dianion for $\beta_{2}$ GPI binding [33]. This particular setting could induce a $\beta_{2}$ GPI configuration enabling sensitive binding of aPL but, obviously, did not provide a reaction environment for the discrimination of aPL occurring in APS and asymptomatic SARD patients. However, quantitative aPL testing did reveal significantly different levels of aCL IgG by LIA like did quantitative aPA, aPS, aPG, and aPT IgG analysis by this method. Of note, quantitative aPS IgM testing did corroborate the significant difference of the qualitative one. In contrast, consensus criteria' aPL IgM analysis by ELISA did not reveal significantly different aPL levels in APS and asymptomatic SARD patients. This further highlights the specificity of the LIA reaction environment for aPL analysis and the putative role of differing $\beta_{2}$ GPI configurations for specific aPL binding. 
Recently, the ratio of aD1 to aD4-5 was reported as a useful marker for APS [9]. Surprisingly, the recommended cutoff of aD1/D4-5 did not enable differentiating APS from SARD patients in this study. Only after applying ROC curve analysis and readjusting the cut-off to 4.6, significantly different prevalences in both groups were determined. Furthermore, the ROC curve analysis of the aD1/aD4-5 ratio revealed the best performance compared with the consensus criteria aPL determined by ELISA.

The assay performance of aPG IgG analysis being the best amongst the aPL detection by LIA was not significantly different from the performance of the aD1/aD4-5 ratio. In addition, there was no significant difference and a good agreement between qualitative aPG IgG analysis by LIA and D1/D4-5 ratio assessment. Thus, the LIA reaction environment consisting of immobilized PG interacting with the specimen's $\beta_{2}$ GPI might favor the specific binding of $\mathrm{aD} 1$ as shown for $\mathrm{aPL}$ IgG testing recently (Fig. 1) [16]. Furthermore, a specific conformation of the bound $\beta_{2}$ GPI as a single molecule or of a $\beta 2$ GPI layer could minimize the binding of $\mathrm{aD} 4-5$ and, thus, bring about the high specificity of aPG analysis. As a fact, aD4-5 did not seem to be associated with the APS phenotype [7]. A similar LIA reaction environment with CL (diphosphatidylglycerol) instead of PG demonstrated a significantly poorer assay performance which underlines the importance of the phospholipid nature for specific aPL binding.

Our study has certain limitations. The relatively low number of samples results in large confidence interval (CI) in particular regarding the comparison of AUC values by ROC analysis. Furthermore, there could be a selection bias for patients with SARD, since these patients were recruited by a $\beta_{2}$ GPI IgG positivity. We employed research ELISA for $\mathrm{aD} 1$ analysis which might differ from the presently available commercial aD1 assays.

In summary, aPG IgG analysis by LIA and assessment of the $\mathrm{aD} 1 / \mathrm{aD} 4-5$ ratio by ELISA enabled the discrimination of aPL in patients with APS from those with asymptomatic SARD. This finding could be helpful in clinical practice, mainly because patients at risk of adverse APS events could be identified and managed more appropriately by primary prophylaxis and more frequent clinical controls [34].

\section{Compliance with ethical standards}

Conflict of interest DR has a management role and is a shareholder of GA Generic Assays GmbH and Medipan GmbH. Both companies are diagnostic manufacturers. MM is employed at Inova Diagnostics selling autoantibody assays. TB is employed at GA Generic Assays selling autoantibody assays. VS is an employee of Medipan. All the other authors declare that they have no financial competing and nonfinancial conflicts of interest.
Human and animal rights The study was approved by the local ethics committee and was conducted in accordance with the Helsinki Declaration of 1964 (revised 2008).

Informed consent We hereby declare that all the patients were examined in the outpatient clinic at the Division of Rheumatology of the University of Brescia in Italy, and they have all consented to donate their sera for the purpose of this publication. Furthermore, this study was approved by the local ethical committee

Open Access This article is distributed under the terms of the Creative Commons Attribution 4.0 International License (http://creativeco mmons.org/licenses/by/4.0/), which permits unrestricted use, distribution, and reproduction in any medium, provided you give appropriate credit to the original author(s) and the source, provide a link to the Creative Commons license, and indicate if changes were made.

\section{References}

1. Ruiz-Irastorza G, Crowther M, Branch W, Khamashta MA (2010) Antiphospholipid syndrome. Lancet 376:1498-1509

2. Meroni PL, Borghi MO, Raschi E, Tedesco F (2011) Pathogenesis of antiphospholipid syndrome: understanding the antibodies. Nat Rev Rheumatol 7:330-339

3. Asherson RA, Cervera R, DeGroot PG, Erkan D, Boffa MC, Piette JC, Kamashta MA, Shoenfeld Y (2003) Catastrophic antiphospholipid syndrome: international consensus statement on classification criteria and treatment guidelines. Lupus 12:530-534

4. Bertolaccini ML, Amengual O, Andreoli L, Atsumi T, Chighizola CB, Forastiero R, deGroot P, Lakos G, Lambert M, Meroni P, Ortel TL, Petri M, Rahman A, Roubey R, Sciascia S, Snyder M, Tebo AE, Tincani A, Willis R (2014) 14th International Congress on Antiphospholipid Antibodies Task Force. Report on antiphospholipid syndrome laboratory diagnostics and trends. Autoimmun Rev 13:917-930

5. Tebo AE, Willis R, Jaskowski TD, Guerra M, Pierangeli SS, Salmon J, Petri M, Branch DW (2016) Clinical significance and correlations between anti-beta2 glycoprotein I IgA assays in antiphospholipid syndrome and/or systemic lupus erythematosus. Clin Chim Acta 460:107-113

6. Nalli C, Piantoni S, Andreoli L, Motta M, Tincani A (2012) Antiphospholipid syndrome and antiphospholipid antibodies in children: the two sides of the coin. Isr Med Assoc J 14:310-312

7. Andreoli L, Nalli C, Motta M, Norman GL, Shums Z, Encabo S, Binder WL, Nuzzo M, Frassi M, Lojacono A, Avcin T, Meroni PL, Tincani A (2011) Anti-beta(2)-glycoprotein I IgG antibodies from 1-year-old healthy children born to mothers with systemic autoimmune diseases preferentially target domain 4/5: might it be the reason for their 'innocent' profile? Ann Rheum Dis 70:380-383

8. deLaat B, Derksen RH, Urbanus RT, DeGroot PG (2005) IgG antibodies that recognize epitope Gly40-Arg43 in domain I of beta 2-glycoprotein I cause LAC, and their presence correlates strongly with thrombosis. Blood 105:1540-1545

9. Andreoli L, Chighizola CB, Nalli C, Gerosa M, Borghi MO, Pregnolato F, Grossi C, Zanola A, Allegri F, Norman GL, Mahler M, Meroni PL, Tincani A (2015) Clinical characterization of antiphospholipid syndrome by detection of $\mathrm{IgG}$ antibodies against beta2-glycoprotein i domain 1 and domain 4/5: ratio of anti-domain 1 to anti-domain $4 / 5$ as a useful new biomarker for antiphospholipid syndrome. Arthritis Rheumatol 67:2196-2204 
10. Mattia E, Ruffatti A, Meneghel L, Tonello M, Faggian D, Hoxha A, Fredrigo M, Punzi L, Plebani M (2015) A contribution to detection of anticardiolipin and anti-beta2glycoprotein I antibodies: comparison between a home-made ELISA and a fluorescence enzyme immunoassay. Clin Chim Acta 446:93-96

11. Mahler M, Norman GL, Meroni PL, Khamashta M (2012) Autoantibodies to domain 1 of beta 2 glycoprotein 1: a promising candidate biomarker for risk management in antiphospholipid syndrome. Autoimmun Rev 12:313-317

12. Fickentscher C, Magorivska I, Janko C, Biermann M, Bilyy R, Nalli C, Tincani A, Medeghini V, Meini A, Nimmerjahn F, Schett G, Muñoz LE, Andreoli L, Herrmann M (2015) The pathogenicity of anti- $\beta 2 \mathrm{GP} 1-\mathrm{IgG}$ autoantibodies depends on Fc glycosylation. J Immunol Res 2015:638129

13. Mahler M, Albesa R, Zohoury N, Bertolaccini ML, Ateka-Barrutia O, Rodriguez-Garcia JL, Norman GL, Khamashta M (2016) Autoantibodies to domain 1 of beta 2 glycoprotein I determined using a novel chemiluminescence immunoassay demonstrate association with thrombosis in patients with antiphospholipid syndrome. Lupus 25:911-916

14. Meneghel L, Ruffatti A, Gavasso S, Meneghel L, Tonello M, Salvan E, Pengo V, Punzi V (2015) Detection of IgG anti-domain I beta2 Glycoprotein I antibodies by chemiluminescence immunoassay in primary antiphospholipid syndrome. Clin Chim Acta 446:201-205

15. Erkan D, Lockshin MD (2012) APS ACTION-antiphospholipid syndrome alliance for clinical trials and international networking. Lupus 21:695-698

16. Roggenbuck D, Borghi MO, Somma V, Büttner T, Schierack P, Hanack K, Grossi C, Bodio C, Macor P, von Landenberg P, Boccellato F, Mahler M, Meroni PL (2016) Antiphospholipid antibodies detected by line immunoassay differentiate among patients with antiphospholipid syndrome, with infections and asymptomatic carriers. Arthritis Res Ther 18:111

17. Meroni PL, Biggioggero M, Pierangeli SS, Sheldon J, Zegers I, Borghi MO (2014) Standardization of autoantibody testing: a paradigm for serology in rheumatic diseases. Nat Rev Rheumatol 10:35-43

18. Pengo V, Biasiolo A, Pegoraro C, Cucchini U, Noventa F, Iliceto S (2005) Antibody profiles for the diagnosis of antiphospholipid syndrome. Thromb Haemost 93:1147-1152

19. Roggenbuck D, Egerer K, von Landenberg P, Hiemann R, Feist E, Burmester GR, Dörner T (2012) Antiphospholipid antibody profiling-time for a new technical approach. Autoimmun Rev $11: 821-826$

20. Hoxha A, Ruffatti A, Mattia E, Meneghel L, Tonello M, Salvan E, Pengo V, Punzi L (2015) Relationship between antiphosphatidylserine/prothrombin and conventional antiphospholipid antibodies in primary antiphospholipid syndrome. Clin Chem Lab Med 53:1265-1270

21. Egerer K, Roggenbuck D, Buettner T, Lehmann B, Kohn A, von Landenberg P, Hiemann R, Feist E, Burmester GR, Dörner T (2011) Single-step autoantibody profiling in antiphospholipid syndrome using a multi-line dot assay. Arthritis Res Ther 13:R118
22. Roggenbuck D, Egerer K, Feist E, Burmester GR, Dorner T (2012) Antiphospholipid antibody profiling-association with the clinical phenotype in APS? Arthritis Rheum 64:2807-2808

23. Roggenbuck D, Somma V, Schierack P, Borghi MO, Meroni PL (2014) Autoantibody profiling in APS. Lupus 23:1262-1264

24. Pengo V (2012) ISTH guidelines on lupus anticoagulant testing. Thromb Res 130(Suppl 1):S76-S77

25. Devreese KM, Pierangeli SS, de Laat Tripodi A, Atsumi T, Ortel TL (2014) Testing for antiphospholipid antibodies with solid phase assays: guidance from the SSC of the ISTH. J Thromb Haemost 12:792-795

26. Miyakis S, Lockshin MD, Atsumi T, Branch DW, Brey RL, Cervera R, Derksen RH, de Groot PG, Koike T, Meroni PL, Reber G, Shoenfeld Y, Tincani A, Vlachoyiannopoulos PG, Krilis SA (2006) International consensus statement on an update of the classification criteria for definite antiphospholipid syndrome (APS). J Thromb Haemost 4:295-306

27. Meroni PL (2016) Anti-beta-2 glycoprotein I epitope specificity: from experimental models to diagnostic tools. Lupus 25:905-910

28. Pengo V, Ruffatti A, Tonello M, Cuffaro S, Banzato A, Bison E, Denas G, Padayattil Jose S (2015) Antiphospholipid syndrome: antibodies to Domain 1 of beta2-glycoprotein 1 correctly classify patients at risk. J Thromb Haemost 13:782-787

29. Pengo V, Banzato A, Denas G, Jose SP, Bison E, Hoxha A, Ruffatti A (2013) Correct laboratory approach to APS diagnosis and monitoring. Autoimmun Rev 12:832-834

30. Schoenherr G, Roggenbuck D, Seifert M, Jahn S, Porstmann T (1996) Technical problems arising from the use of the immunoblot for determination of the reactivity of natural antibodies with different lipopolysaccharides (LPS). J Immunol Methods 190:185-188

31. Seifert M, Schoenherr G, Roggenbuck D, Marx U, von Baehr $\mathrm{R}$ (1996) Generation and characterization of a human monoclonal IgM antibody that recognizes a conserved epitope shared by lipopolysaccharides of different gram-negative bacteria. Hybridoma 15:191-198

32. Conrad K, Schneider H, Ziemssen T, Talaska T, Reinhold D, Humbel RL, Roggenbuck D (2007) A new line immunoassay for the multiparametric detection of antiganglioside autoantibodies in patients with autoimmune peripheral neuropathies. Ann N Y Acad Sci 1109:256-264

33. Sathappa M, Alder NN (2016) The ionization properties of cardiolipin and its variants in model bilayers. Biochim Biophys Acta 1858:1362-1372

34. Chighizola CB, Ubiali T, Meroni PL (2015) Treatment of thrombotic antiphospholipid syndrome: the rationale of current management - an insight into future approaches. J Immunol Res 2015:951424

Publisher's Note Springer Nature remains neutral with regard to jurisdictional claims in published maps and institutional affiliations.

\section{Affiliations}

\section{Cecilia Nalli ${ }^{1}$ Valentina Somma ${ }^{2} \cdot$ Laura Andreoli $^{1} \cdot$ Thomas Büttner $^{3} \cdot$ Peter Schierack $^{4} \cdot$ Michael Mahler $^{5}$. Dirk Roggenbuck ${ }^{2,4}$ (1) . Angela Tincani ${ }^{1}$}

Cecilia Nalli

cecilianalli2@gmail.com
Valentina Somma

v.somma@medipan.de 
Laura Andreoli

laura.andreoli@unibs.it

Thomas Büttner

thomas.buettner@genericassays.com

Peter Schierack

peter.schierack@b-tu.de

Michael Mahler

mmahler@inovadx.com

Angela Tincani

angela.tincani@unibs.it
1 University of Brescia, Brescia, Italy

2 Research and Development Department, Medipan GmbH, Dahlewitz, Berlin, Germany

3 Research and Development Department, GA Generic Assays $\mathrm{GmbH}$, Dahlewitz, Berlin, Germany

4 Institute of Biotechnology, Faculty Environment and Natural Sciences, Brandenburg University of Technology

Cottbus-Senftenberg, Universitätsplatz 1, 01968 Senftenberg, Germany

5 Inova Diagnostics, San Diego, CA, USA 